\title{
HEART-RATE VARIABILITY IN WOMEN DURING 40-HOUR PROLONGED WAKEFULNESS
}

\author{
Doreen Anders, ${ }^{1}$ Stephanie Vollenweider, ${ }^{1}$ Judith Cann, ${ }^{1}$ \\ Marcel Hofstetter, ${ }^{1}$ Josef Flammer, ${ }^{2}$ Selim Orgül, ${ }^{2}$ and Kurt Kräuchi ${ }^{1}$ \\ ${ }^{1}$ Thermophysiological Chronobiology, Centre for Chronobiology, Psychiatric Hospital of the \\ University of Basel, Basel, Switzerland \\ ${ }^{2}$ University Eye Clinic, Basel, Switzerland
}

\begin{abstract}
Heart-rate variability patterns of 18 women during a 40-h constant routine of prolonged wakefulness under controlled laboratory conditions were analyzed. The authors tested the circadian timing of the autonomic nervous system and the relationship between the sympathetic and vagal branches in women with both a functional disorder of vascular regulation (main symptom: cold hands and feet) and prolonged sleep onset and controls without these symptoms. Spectral analysis of R-R intervals during paced breathing episodes revealed significantly lower power values in the high-frequency band (HF; $0.15-0.4 \mathrm{~Hz}$ ) but not in the low-frequency band (LF; $0.04-0.15 \mathrm{~Hz}$ ), leading to a significantly elevated LF/HF ratio in the former group. A significant circadian rhythm in LF power and heart rate occurred in both groups, and a significant correlation was found between sleepiness and sympathovagal balance $(r=.53, p<.05)$. These findings indicate not only an autonomic imbalance in the first group compared with controls, but also two strategies of the autonomic nervous system to fight against fatigue in women. One implies circadian control and the other homeostatic control, and both are reflected by the LF/HF ratio. (Author correspondence: Kurt.Kraeuchi@upkbs.ch)
\end{abstract}

Keywords Autonomic nervous system; Blood pressure; Circadian rhythms; Constant routine; Heart rate variability; Paced breathing; Vascular dysregulation

\section{INTRODUCTION}

Women with symptoms of both primary vascular dysregulation (PVD) and difficulties initiating sleep (DIS) have a mismatched internal phase of entrainment (Vollenweider et al., 2008). In these women, core body

Submitted April 1, 2010, Returned for revision May 4, 2010, Accepted June 16, 2010

Address correspondence to Kurt Kräuchi, Thermophysiological Chronobiology, Centre for Chronobiology, Psychiatric Hospital of the University of Basel Wilhelm Klein Strasse 27, 4012 Basel, Switzerland. Tel: +41 61325 5508; Fax: +4161325 5556; E-mail: Kurt.Kraeuchi@upkbs.ch 
temperature and melatonin rhythms, validated markers of the internal clock, are phase-delayed compared with healthy controls (CON). The main physiological underpinning of PVD is the dysregulation of blood vessels. Under specific stimuli, such as cold, these individuals exhibit a contraction of acral vessels, which results in cold extremities (Flammer et al., 2001). Given that the vasculature in the skin is controlled by the autonomic nervous system (ANS), the question arises as to whether women with PVD and DIS (WVD) display a different pattern in the parasympathetic and sympathetic balance underlying cardiac control.

Within the framework of cardiac autonomic activity, heart-rate variability (HRV) is a well-known tool that provides a window onto autonomic modulation of the heart through frequency domain parameters that can be used to accurately interpret sympathovagal balance (Narita et al., 2007; Pagani et al., 1986; Pomeranz et al., 1985; Task Force of the European Society of Cardiology and the North America Society of Pacing Electrophysiology, 1996). Studies focusing on pharmacological interventions have revealed that blocking the muscarinic parasympathetic transmission can abolish frequency peaks around $0.12-0.4 \mathrm{~Hz}$ and reduce the peak at $0.04 \mathrm{~Hz}$ in the power spectrum of heart-rate (HR) fluctuations (Akselrod et al., 1981). Beta-sympathetic blockade, combined with raised arterial pressure, increased the peak at around $0.04 \mathrm{~Hz}$ (Akselrod et al., 1981). Taken together, this suggests that in HRV spectra the lowfrequency band (LF; 0.04-0.15 Hz) mirrors the influence of both sympathetic and parasympathetic activities, the high-frequency band (HF; $0.15-0.4 \mathrm{~Hz}$ ) is associated with parasympathetic activity, and the LF/HF ratio is indicative of sympathovagal balance (Task Force of the European Society of Cardiology and the North America Society of Pacing Electrophysiology, 1996).

However, there still is an open debate as to the functional significance of the very low frequency (VLF) band of HRV. The VLF band at $0.0033-0.04 \mathrm{~Hz}$ reflects even slower modulations of HR, and may represent the influence of the peripheral vasomotor and renin-angiotensin systems (Akselrod et al., 1981). It is also likely that VLF power is influenced by thermal stimuli (Fleisher et al., 1996). If this, indeed, is the case, one might expect for WVD to show differences to controls in this frequency domain.

Breathing plays a pivotal role on HRV output, particularly in the high-frequency domain. Respiration shows a circadian modulation with troughs of respiratory variables at $6-8 \mathrm{~h}$ before the core body temperature minimum (Spengler et al., 2000). Furthermore, a higher power in the HF band occurs with increased respiratory rate, resulting in maximal power values for 12 breaths/min (Guzik et al., 2007). Only under fixed-frequency breathing is it possible to make a clear distinction between LF and HF oscillations (Cooke et al., 1998). 
There is evidence of the clustering of cardiac events between 06:00 and 12:00 $\mathrm{h}$ in the morning (after habitual nocturnal sleep) in a subset of arrhythmic patients (Goldstein et al., 1996; Gupta \& Shetty, 2005; Marsh et al., 1990; Muller, 1999). Whereas the time course of HRV during a normal night's sleep is fairly well known, it remains controversial as to whether the endogenous circadian rhythmicity of the sympathetic and parasympathetic nervous systems exhibits a similar profile when people are sleep-deprived. Thus, we investigated the endogenous circadian rhythm of HRV parameters to examine how and in what direction the relationship between the sympathetic and vagal branches and/or the timing of the ANS between WVD and CON change during sleep deprivation under controlled constant-routine laboratory conditions.

\section{MATERIALS AND METHODS}

\section{Subjects}

Eighteen healthy women (9 CON and 9 WVD) were selected to have had no shiftwork within 3 months or transatlantic flight within 1 month prior to the study, no medication (including hormones) or drug abuse, and be nonsmokers. Further exclusion criteria were extreme morning or evening chronotypes, amenorrhea, or an irregular menstrual cycle. All volunteers were given questionnaires and a medical examination to determine physiological and psychological health. They completed a stringently controlled 40-h constant-routine (CR) protocol of prolonged wakefulness during the luteal phase. Chronotype was estimated using the morning-evening-type questionnaire of Torsvall and Åkerstedt (1980).

The experimental protocol was approved by the local ethical committee (Human Research Committee of the Department of Medicine, University of Basel) and conformed with the ethical standards of the journal (Portaluppi et al., 2008). The main purpose and risks of the study were explained to the subjects before they gave their written consent. They could break off the experiment at any time. All subjects completed the study without any complaints.

Because there is strong body of evidence that acral skin temperature and skin temperature gradient are correlated with blood flow (House \& Tipton, 2002; Rubinstein et al., 1990) or vasomotion (Severens et al., 2010), the screening for CON or WVD was not only conducted using subjective information about thermal discomfort with cold extremities (TDC), but also by finger temperature. Difference in vasomotion between CON and WVD was confirmed by the measurement of the distal-proximal skin temperature gradient before the baseline night. Additionally finger nailfold video capillary microscopy for objective assessments was carried out (criteria: blood standstill for $\geq 12 \mathrm{~s}=$ women with PVD, $<12 \mathrm{~s}=\mathrm{CON}$ ) 
(Emre et al., 2004; Gasser et al., 1991). DIS was evaluated by questionnaires, as well as via polysomnography during a screening night.

\section{Design and Procedure}

Within the screening procedure each woman spent a night in the sleep laboratory to confirm that they were able to sleep in the laboratory and to document whether the electroencephalogram (EEG) and electrocardiogram (ECG) patterns were normal.

One week before the experimental protocol began, subjects were requested to maintain a sleep-wake cycle according to their habitual bedtime and to refrain from caffeine, alcohol, or extensive exercise $(<5 \mathrm{~h} / \mathrm{wk})$. Subjects came to the laboratory $2 \mathrm{~h}$ before their habitual bedtime for an adaption night. They were prepared for sleep with ECG (V2, V5), EEG (C3,C4, Cz, Pz, Oz, Fz), EMG (chin), and electrooculogram (EOG; outer canthi) electrodes, as well as a rectal probe (polyoxymethylene probe: 2-mm diameter, copper-constantan, Interstar, Cham, Switzerland; Therm, type 5500-3, Ahlborn, Holzkirchen, Germany) and eight skin temperature probes (silver disk: 1-cm diameter, copper-constantan, model P224, Prof. Schwamm, Ahlborn; Therm type 5500-3, Ahlborn, Holzkirchen, Germany). After awakening the subjects on the following day, the time until start of the protocol was used to adjust them to the experimental dim light conditions ( $<8$ lux). They were allowed to walk around the laboratory, but they had to wear sunglasses when walking out of the dimmed room to assure no strong light input.

Individual protocols started in the afternoon, $8 \mathrm{~h}$ before usual bedtime; the subsequent night was the baseline night followed by an adapted (Mills et al., 1978) 40-h CR protocol and a recovery night. Participants remained in dim-light conditions $(<8$ lux during wakefulness/0 lux during sleep) under a constant semirecumbent posture position in bed (head up not more than $45^{\circ}$ during wakefulness/bed position $0^{\circ}$ during sleep). Room temperature and relative humidity were kept constant at $22^{\circ} \mathrm{C}$ and $55 \%$, respectively. Subjects were studied singly, insulated from external sound or time cues; isocaloric sandwiches and water were administered at hourly intervals; non-overly stimulating neuropsychological tests were carried out every $2 \mathrm{~h}$; and saliva and selfratings of sleepiness (Karolinska Sleepiness Scale; KSS) were collected at half-hourly intervals. Blood pressure (BP) measurements were taken every hour (ambulatory blood pressure monitor; MOBIL-O-GRAPH ${ }^{\circledR}$ Vers. 12; I.E.M., Stolberg, Germany).

In order to control breathing frequency, paced breathing (12 breaths/ $\min =0.2 \mathrm{~Hz}$ ) for a duration of $3 \mathrm{~min}(20$ paced breathing episodes $/ 40 \mathrm{~h}$ ) was carried out every $2 \mathrm{~h}$ throughout the CR protocol. During the paced breathing episode, subjects were instructed to inhale and exhale naturally 
according to regularly 5-s auditory signals (spoken voice recorded and rendered via a computer mediaplayer; inhale:exhale $=2: 3 \mathrm{~s}$ ). Furthermore, each power spectrum was checked whether a peak in log power of HRV occurred at $0.2 \mathrm{~Hz}$. During the breathing episodes, subjects carried out the Karolinska Drowsiness Test, where movements are forbidden.

\section{Data Acquisition and Data Analysis}

Standard Ag-AgCl surface electrodes were placed on V2 and V5 (modified precordial lead). ECG was recorded with the VITAPORT ambulatory system (Vitaport-3 digital recorder; TEMEC Instruments, Kerkrade, The Netherlands) with a sampling rate of $512 \mathrm{~Hz}$. The raw signals were stored online on a Flash RAM card (Viking) and later downloaded off-line to a PC hard drive. A computerized system (System Hofstetter ${ }^{\circledR}$; SHS Allschwil) was used to analyze the signals and detect the length of all R-R intervals over the entire 40-h CR, in particular for the $20 \times 3$-min paced breathing episodes. Each output was checked for plausibility by visual inspection. Obviously missing R-peak detections were replaced by interpolation of surrounding data, and all R-R peak durations $<0.3 \mathrm{~s}$ and $>1.8 \mathrm{~s}$ were eliminated. If the noise was low, this time duration was taken for further HRV analyses. HR was calculated as $1 /$ median $\times 60$ for all paced 3 -min sequences.

Spectral analysis of all 3-min sequences of R-R intervals were performed by a fast Fourier transform algorithm tapered with a Parzen window, after equidistant resampling with $16 \mathrm{~Hz}$. Data were bidirectionally smoothed by a low-pass filter with a cut-off frequency of $0.8 \mathrm{~Hz}$. The power for VLF, LF, and HF was calculated by the power spectrum. The $\mathrm{LF} / \mathrm{HF}$ ratio was taken as a measure for the balance of the ANS.

Rhythm analyses were performed separately for both groups with ChronosFit (Zuther et al., 2009). This program combines a partial Fourier analysis and a stepwise regression technique. It fits the harmonics separately and carries out the $F$ test for each. Afterwards the most significant harmonic is included in the model. Additionally, nonorthogonal spectral analysis was used to fit the data to a two-harmonic model without correlated noise (Brown \& Czeisler, 1992). Phases between 23 and $25 \mathrm{~h}$ were taken into account.

\section{Statistics}

Spectral power indices were logarithmically transformed to correct for a non-Gaussian distribution. The statistical packages Statview ${ }^{\mathrm{TM}}$ 5.0 (SAS Institute, Cary, NC, USA) and Statistica ${ }^{\mathrm{TM}} 6$ for Windows (StatSoft, Tulsa, OK, USA) were used. Time-course analyses were performed using twoway analysis of variance (ANOVA) for repeated measures (rANOVA). The 
grouping factor was "group" (WVD versus $\mathrm{CON}$ ), and the independent variable was the factor time. Following rANOVA, all $p$ values were based on Huyhn-Feldt-corrected degrees of freedom (reported are original degrees of freedom). Fisher's protected least-significant differences procedure with alpha-correction for multiple comparisons (Curran-Everett, 2000) were calculated for post-hoc comparisons. For statistical analyses between WVD and CON without an a priori hypothesis, the threshold for alpha-errors was set at $p<.05$ (two-sided). The Mann-Whitney $U$ test was used to reveal significant differences between WVD and CON.

Linear regression analysis for comparing slopes of $\mathrm{BP}$ and $\mathrm{HF}$ power was performed. To examine the correlation of KSS values and $\log \mathrm{LF} /$ HF ratio, data were pooled. A multiple linear regression analysis with repeated measures was accomplished. The between-subject differences were taken into account using dummy-coded subjects as forced variables in the model (Slinker \& Glantz, 2008).

SigmaPlot ${ }^{\circledR}$ (Systat Software, Chicago, IL, USA) was used for graphics, whereby values are presented as means \pm standard errors (SE).

\section{RESULTS}

\section{Demographic Data and Physiological Characteristics}

Groups were matched for age, body mass index, and chronotype $(p>.1$; Table 1). WVD had objectively measured significantly lower finger temperatures than $\mathrm{CON}\left(28.5^{\circ} \mathrm{C} \pm 0.99^{\circ} \mathrm{C}\right.$ versus $32.83^{\circ} \mathrm{C} \pm$ $0.49^{\circ} \mathrm{C} ; p<.05$ ) and prolonged sleep onset (self-rated: $31.59 \pm 4.46$ versus $15.02 \pm 3.27 \mathrm{~min}$; PSG: $37.41 \pm 10.47$ versus $10.04 \pm 1.14 \mathrm{~min}$; $p<.05)$. Distal-proximal skin temperature gradient was significantly decreased in WVD compared to CON (mean of $8 \mathrm{~h}$ before baseline night: $-3.08^{\circ} \mathrm{C} \pm 0.37^{\circ} \mathrm{C}$ versus $\left.-1.87^{\circ} \mathrm{C} \pm 0.24^{\circ} \mathrm{C} ; p<.05\right)$.

\section{Heart Rate and Heart-Rate Variability}

In Figure 1 spectral analysis data are shown for all frequency bands. In WVD, paced breathing data revealed significantly lower log power

TABLE 1 Subject's demographic data and habitual bed times

\begin{tabular}{llllcc}
\hline Variable & Age $(\mathrm{yr})$ & BMI $\left(\mathrm{kg} / \mathrm{m}^{2}\right)$ & Chronotype & $\begin{array}{c}\text { Habitual light-off times } \\
\text { (clock time) }\end{array}$ & $\begin{array}{c}\text { Habitual awakening } \\
\text { times (clock time) }\end{array}$ \\
\hline WVD & $24.2 \pm 1.2$ & $20.82 \pm 0.54$ & $16.0 \pm 1.6$ & $23: 25 \pm 0: 12$ & $07: 24 \pm 0: 12$ \\
CON & $25.1 \pm 1.7$ & $20.85 \pm 0.6$ & $16.7 \pm 0.9$ & $23: 46 \pm 0: 07$ & $07: 44 \pm 0: 07$ \\
\hline
\end{tabular}

Values are means $\pm \mathrm{SE}$. WVD $=$ women with both primary vascular dysregulation and difficulties initiating sleep; $\mathrm{CON}=$ controls; $\mathrm{BMI}=$ body mass index. 


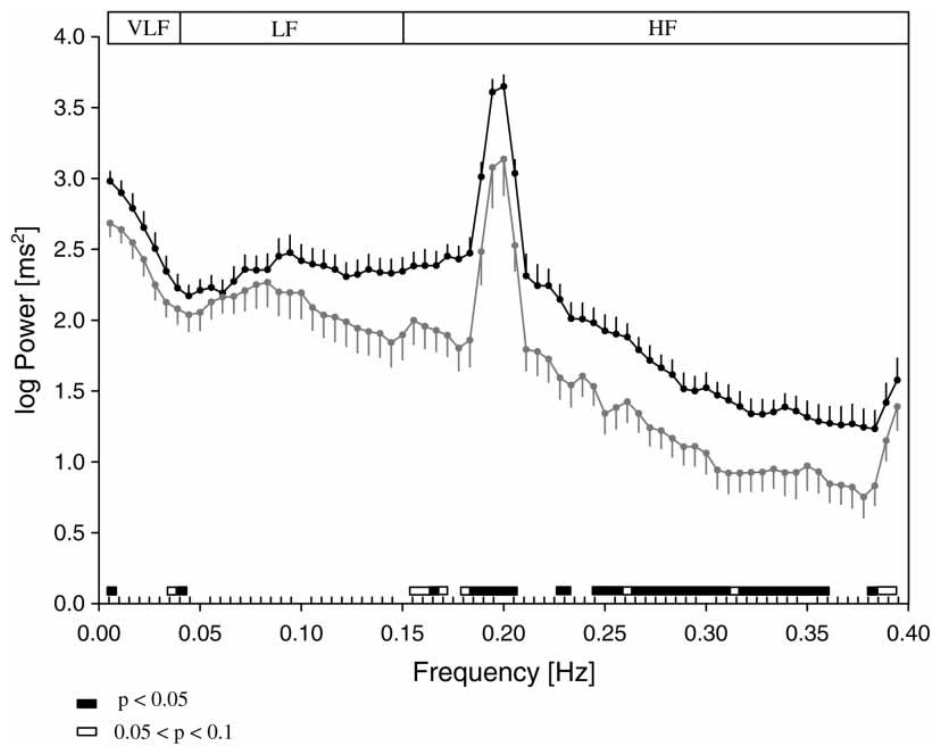

FIGURE 1 Power spectrum of heart rate variability. Data were averaged per constant-routine episode for each subject before averaging across subjects (log mean $\pm \mathrm{SE}, \mathrm{N}=9$ each group; black line $=\mathrm{CON}$; grey line $=\mathrm{WVD})$. Horizontal black bars represent significant differences between WVD and CON $(p<.05$; open bars, $p<.1)$. The very low frequency (VLF) band includes frequencies between 0.0033 and $0.04 \mathrm{~Hz}$, the low-frequency (LF) band frequencies between 0.04 and $0.15 \mathrm{~Hz}$, and the high-frequency (HF) band frequencies between 0.15 and $0.4 \mathrm{~Hz}$.

values, especially in HF, but not in LF or VLF (except frequency band at 0.0056 and $0.0444 \mathrm{~Hz}$ ), with a breathing peak at $0.2 \mathrm{~Hz}$.

The time course of the HR and HRV parameters over the whole CR are shown in Figure 2. Significant differences between WVD and CON occurred in the HF band and the ratio of LF/HF. A tendency towards differences was found in the VLF band. The factor time was significantly different for HR, LF band, HF band, and LF/HF ratio. No significant interaction term between $\mathrm{WVD} / \mathrm{CON}$ and time-of-day was found in HR or any HRV frequency band (Table 2). HR seems to be higher on the second day of the 40-h CR, but this could be ruled out by rANOVA comparing the first day of the CR (D1) with the second day of the CR (D2; $p=.13)$. HR falls in the subjective night $(\mathrm{sN})$ by about $6 \mathrm{bpm}$ in CON and $3 \mathrm{bpm}$ in WVD (peak of group values of D1 to trough of group values of $\mathrm{sN}$ ).

\section{Blood Pressure}

The time course of systolic blood pressure (SBP), diastolic blood pressure (DBP), mean arterial pressure (MAP), and the difference of SBP and DBP are plotted over the 40-h CR in Figure 3. The factor time was 


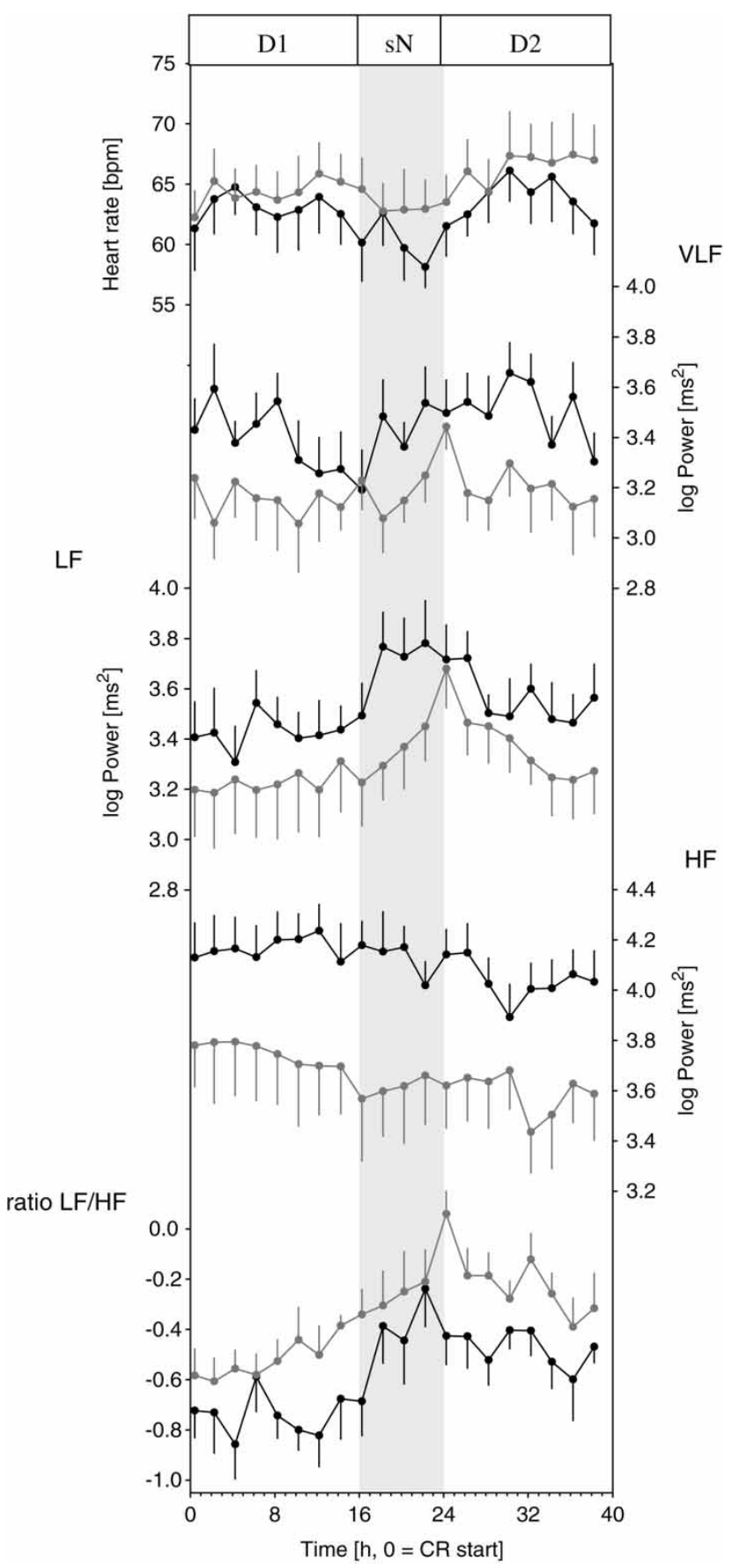

FIGURE 2 Time course (from top to bottom) of heart rate, log-transformed power of very low frequency (VLF; 0.0033-0.04 Hz), low-frequency (LF; 0.04-0.15), and high-frequency (HF; $0.15-0.4 \mathrm{~Hz}$ ) bands, and the $\mathrm{LF} / \mathrm{HF}$ ratio during the constant-routine (CR) with $40 \mathrm{~h}$ of prolonged wakefulness. D1 = first day of CR; $\mathrm{sN}=$ subjective night (grey bar); D2 = second day of CR. (mean \pm $\mathrm{SE}$, black line $=\mathrm{CON}$, grey line $=\mathrm{WVD}, \mathrm{N}=9$ each group.) 
TABLE 2 ANOVA table for HR and HRV variables

\begin{tabular}{|c|c|c|c|c|c|c|}
\hline \multirow{2}{*}{$\begin{array}{l}\text { Variable } \\
\mathrm{HR}\end{array}$} & \multicolumn{2}{|c|}{ Group $(d f=1,16)$} & \multicolumn{2}{|c|}{ Time $(d f=19,304)$} & \multicolumn{2}{|c|}{$\begin{array}{l}\text { Time } \times \text { Group } \\
(d f=19,304)\end{array}$} \\
\hline & $F=0.39$ & $p>.1$ & $F=3.52$ & $p<.05$ & $F=0.92$ & $p>.1$ \\
\hline VLF & $F=4.23$ & $p=.056$ & $F=1.17$ & $p>.1$ & $F=0.92$ & $p>.1$ \\
\hline $\mathrm{LF}$ & $F=1.67$ & $p>.1$ & $F=3.22$ & $p<.05$ & $F=0.72$ & $p>.1$ \\
\hline $\mathrm{HF}$ & $F=4.62$ & $p<.05$ & $F=2.53$ & $p<.05$ & $F=1.04$ & $p>.1$ \\
\hline $\mathrm{LF} / \mathrm{HF}$ & $F=5.27$ & $p<.05$ & $F=5.91$ & $p<.05$ & $F=0.79$ & $p>.1$ \\
\hline
\end{tabular}

Two-way rANOVAs with factors group (CON versus WVD) and time (20 paced breathing episodes during CR) are shown for heart rate (HR), very low frequency power (VLF), low-frequency power (LF), high-frequency power (HF), and the sympathovagal balance (LF/HF).

significantly different for DBP, but no significant difference in group or interaction term between $\mathrm{WVD} / \mathrm{CON}$ and time-of-day was found in any BP variable (Table 3 ).

\section{Rhythm Analysis}

A pooled rhythm analysis was performed due to the fact that no significant interaction term between group and time occurred in any parameter (Figure 4).

ANOVA for the factor time revealed significance for HR, LF band, $\mathrm{HF}$ band, and LF/HF ratio. Within the paced breathing protocol, a circadian rhythm was observed in each of these parameters, except for the HF band (Table 4). The circadian rhythm of the mean HR shows a trough during the middle of $\mathrm{sN}$, between 19.41 and $22.29 \mathrm{~h}$ after $\mathrm{CR}$ start. A broad maximum is seen in the afternoon. Maxima for circadian HRV parameters occur in the latter part of $\mathrm{sN}$, and minima are located in the late evening.

Not all subjects had a significant amplitude for the rhythm analysis and thus no circadian rhythm, but the mean of the fitted minima and maxima of individual CRs with significant amplitude after rhythm analyses are located in the similar time period. No significant circadian rhythm, but significance over time occurred in the HF band. The pooled data of CON and WVD exhibit in the HF band a linear trend over time $(p<.05, F=12.55, d f=1,16)$. Despite the fact that $\log$ ratio of $\mathrm{LF} / \mathrm{HF}$ exhibited a circadian rhythm, the same linear trend is evident $(p<.05$, $F=30.92, d f=1,16)$.

ANOVA for factor time revealed significance only for DBP. No nocturnal decline in BP was seen as found during sleep, but a linear trend with increasing slope for DBP was detected $(p<.05, F=9.04, d f=1,14)$. 


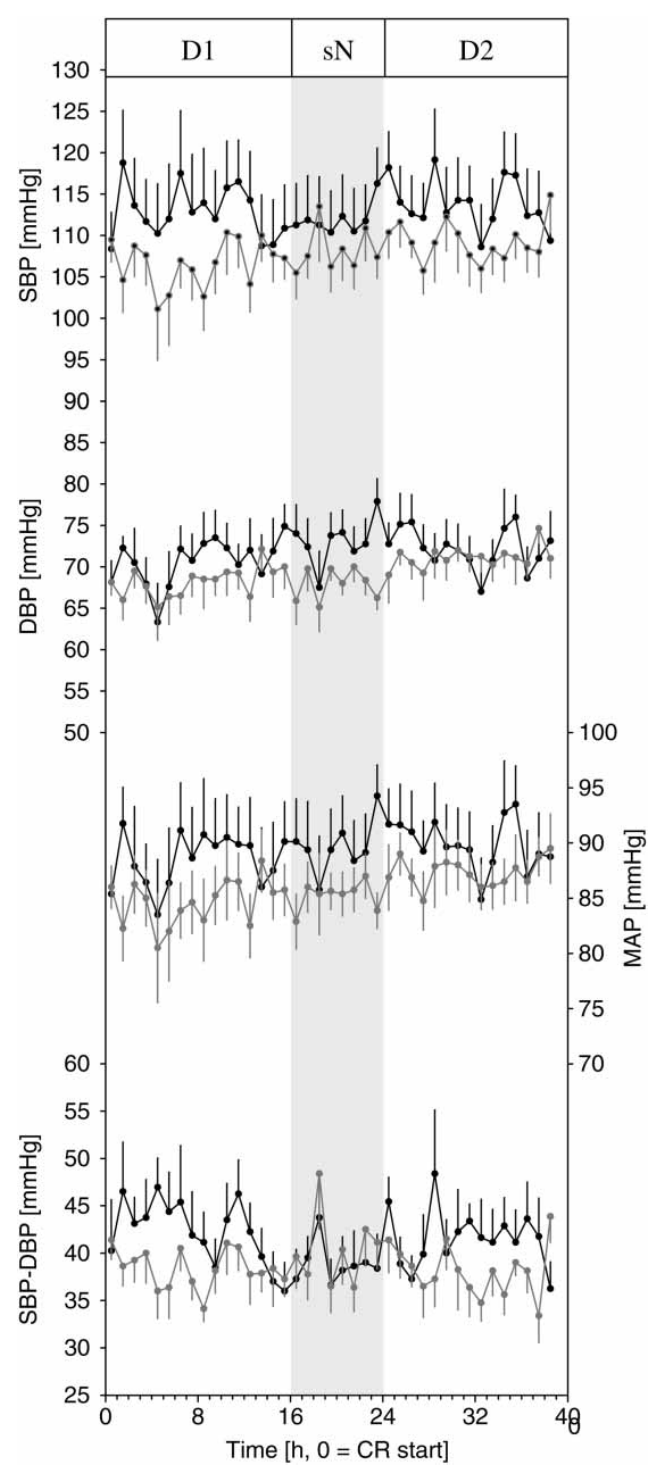

FIGURE 3 Time course of hourly measured blood pressure values during constant-routine (CR) with $40 \mathrm{~h}$ of prolonged wakefulness. D1 = first day of CR; $\mathrm{sN}=$ subjective night (grey bar); D2= second day of CR. From top to bottom: systolic blood pressure (SBP), diastolic blood pressure (DBP), mean arterial pressure (MAP), pulse pressure ( $\mathrm{SBP}-\mathrm{DBP}$; mean $\pm \mathrm{SE})$. Black line $=\mathrm{CON}$; grey line $=\mathrm{WVD}(\mathrm{N}=8$ each group $)$

\section{DISCUSSION}

The present study is the first to examine differences in the ANS modulation of the heart between WVD and CON using HRV analysis. In order to control for interfering factors that might affect HRV, short-term (3-min) ECG recordings were performed during the Karolinska 
TABLE 3 ANOVA table for blood pressure variables

\begin{tabular}{|c|c|c|c|c|c|c|}
\hline \multirow{2}{*}{$\begin{array}{l}\text { Variable } \\
\text { SBP }\end{array}$} & \multicolumn{2}{|c|}{ Group $(d f=1,14)$} & \multicolumn{2}{|c|}{ Time $(d f=38,532)$} & \multicolumn{2}{|c|}{$\begin{array}{l}\text { Time } \times \text { Group } \\
(d f=38,532)\end{array}$} \\
\hline & $F=0.89$ & $p>.1$ & $F=1.16$ & $p>.1$ & $F=1.12$ & $p>.1$ \\
\hline $\mathrm{DBP}$ & $F=0.71$ & $p>.1$ & $F=1.68$ & $p<.05$ & $F=1.15$ & $p>.1$ \\
\hline MAP & $F=0.80$ & $p>.1$ & $F=1.38$ & $p>.1$ & $F=0.98$ & $p>.1$ \\
\hline SBP-DBP & $F=0.80$ & $p>.1$ & $F=1.25$ & $p>.1$ & $F=1.36$ & $p>.1$ \\
\hline
\end{tabular}

Two-way rANOVAs with factors group (CON versus WVD) and time (39 timepoints during CR) for systolic blood pressure (SBP), diastolic blood pressure (DBP), mean arterial pressure (MAP), and pulse pressure (SBP - DBP).

Drowsiness Test (Akerstedt \& Gillberg, 1990) during a 40-h CR protocol of prolonged wakefulness. Using this protocol, environmental and physical conditions were kept constant and emotional as well as sensory stimuli were reduced to a minimum.

The key finding of this study is that HRV spectral analysis revealed specific differences between CON and WVD. During the course of the $40 \mathrm{~h}$ of wakefulness, WVD exhibited lower HF power and higher sympathovagal balance (LF/HF), with no differences in LF power, HR, and BP parameters. In addition, significant circadian patterns were found selectively in HR and LF power; HR, as previously found (Kräuchi \& Wirz-Justice, 1994), was attenuated, and LF power increased during the subjective night. Interestingly, these circadian variations did not differ between CON and WVD. BP (SBP, DBP, and MAP) did not show any circadian variation. However, over the $40 \mathrm{~h}$ of prolonged wakefulness, a linear decrease occurred in the vagal branch of the ANS, and conversely DBP increased in a linear manner. The correlation between sleepiness and sympathovagal balance, which implies active regulation of the ANS against fatigue, is remarkable.

What are the theoretical underpinnings that can account for these specific group differences? WVD subjects were selected on the basis of thermal discomfort with cold extremities (TDC) and difficulties initiating sleep (DIS). Therefore, first it is discussed if TDC influences HRV and second the relationship between DIS and HRV. Finally, the circadian modulation of HRV and changes with increased elapsed time awake and supine position are considered.

\section{How Does TDC Impact on HRV?}

Several studies indicate that the thermal discomfort induced by cool environmental stimuli leads to changes in the HRV pattern (Liu et al., 2008; Nagashima et al., 2002; Yao et al., 2008). Vasoconstriction due to cool ambient temperature is mainly initiated from efferent skin 


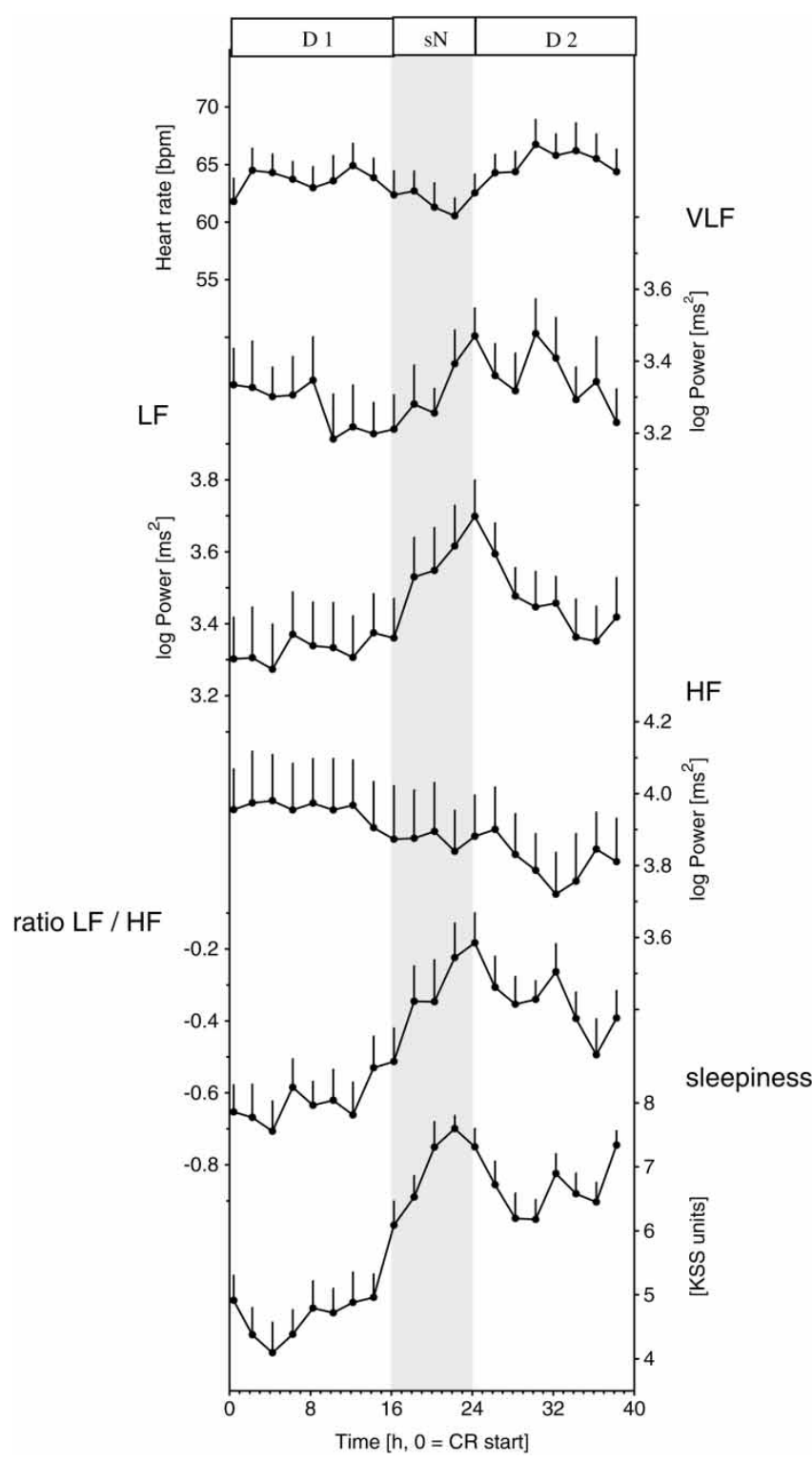

FIGURE 4 Time course of heart rate variables and sleepiness (pooled data, $\mathrm{N}=18$ ) during constant-routine $(\mathrm{CR})$ with $40 \mathrm{~h}$ of prolonged wakefulness. D1 = first day of CR; $\mathrm{sN}=$ subjective night (grey bar); D2 = second day of CR. From top to bottom: heart rate, log-transformed power of very low frequency (VLF), low-frequency (LF), and high-frequency (HF) bands, LF/HF ratio, and subjective ratings of sleepiness on the Karolinska sleepiness scale (KSS; mean $\pm \mathrm{SE}$ ).

sympathetic nerve activity resulting in a higher LF/HF ratio (Liu et al., 2008; Yao et al., 2008). Similarly, WVD in the present sample exhibit a higher LF/HF ratio compared to CON. Ambulatory and controlled laboratory studies have shown that WVD exhibit not only TDC, but also 
TABLE 4 Rhythm analyses

\begin{tabular}{lccccc}
\hline & $\begin{array}{c}\text { Fitted maximum of } \\
\text { mean data with 95 } \\
\text { \% CI (h after CR } \\
\text { start) }\end{array}$ & $\begin{array}{c}\text { Fitted minimum of } \\
\text { mean data with 95 } \\
\text { \% CI h after CR } \\
\text { start) }\end{array}$ & $\begin{array}{c}\text { Subjects } \\
\text { with } \\
\text { significant } \\
\text { amplitude }\end{array}$ & $\begin{array}{c}\text { Mean fitted } \\
\text { maxima of } \\
\text { individual CR } \\
\text { (h after CR } \\
\text { start) } \pm \text { SE (h) }\end{array}$ & $\begin{array}{c}\text { Mean fitted } \\
\text { minima of } \\
\text { individual CR } \\
\text { (h after CR } \\
\text { start) } \pm \text { SE (h) }\end{array}$ \\
\hline HR & $6.12(-0.08-12.31)$ & $20.85(19.41-22.29)$ & $14 / 18$ & $10.25 \pm 1.17$ & $20.90 \pm 0.50$ \\
LF & $21.59(19.42-23.77)$ & $13.19(5.86-20.52)$ & $14 / 18$ & $23.17 \pm 0.94$ & $12.32 \pm 1.05$ \\
HF & n.s. & - & - & - \\
LF/HF & $21.05(16.73-25.40)$ & $13.44(4.45-22.43)$ & $12 / 18$ & $21.60 \pm 0.74$ & $12.29 \pm 0.79$ \\
\hline
\end{tabular}

The table includes fitted maxima and minima of averaged data with $95 \%$ confidence intervals (CIs; hours after constant routine start), subjects with a significant amplitude, and averaged fitted maxima and minima of individual curves with standard error of the mean (SE) for heart rate (HR), lowfrequency power (LF), high-frequency power (HF), and the sympathovagal balance (LF/HF). No significant (n.s.) amplitude for HF could be observed.

objectively measured lower distal skin temperatures (Gompper, 2007; Vollenweider et al., 2008). Thus, from a thermophysiological point of view, WVD live under a more vasoconstricted state in distal skin regions, which could be directly related to the higher sympathovagal balance.

Diminished perfusion of the distal vasculature in WVD leads to a proximal centralization of blood volume. Therefore, heat content remains in the body core (proximal skin temperature and core body temperature are elevated in WVD compared to $\mathrm{CON}$; recalculation of published data in Vollenweider et al., 2008). After bathing in warm water $\left(39^{\circ} \mathrm{C}\right), \mathrm{HF}$ power decreases in CON (Kräuchi, unpublished data). Other studies have also shown attenuation of vagal activity when body heat content was elevated (Brenner et al., 1997; Bruce-Low et al., 2006). Thus, one might argue that changes in WVD thermoregulatory activity is associated with withdrawal of the vagal branch of the ANS.

An additional link between TDC and a different HRV pattern in WVD could evolve from a psychological point of view. Many emotional disorders (related to anxiety, fear, suppressed anger, panic, etc.) have been associated with a reduction in HF power (Alvarenga et al., 2008; Bleil et al., 2008; Cohen et al., 2000; Friedman \& Thayer, 1998a, 1998b; Johnsen et al., 2003; Klein et al., 1995; Marci et al., 2007; Ottaviani et al., 2009). A recently published study shows that a selective suppression of experienced anger was associated with TDC and increased sleep onset latency in women (Von Arb et al., 2009). Both TDC and increased sleep onset latency were part of the inclusion criteria for WVD in the present study as well. Although anger suppression was not measured in the present sample, it might explain the relationship between psychological conditions and HRV. Therefore, prospective studies are needed to show whether anger suppression can explain the reduced HF power in WVD. 


\section{What is the Role of DIS on HRV?}

TDC in WVD seems to contribute to the decrease in the HF band, but does DIS also have an impact on HRV spectrum? DIS is one of the Diagnostic and Statistical Manual of Mental Disorders Fourth Edition (DSM-IV) criteria for primary insomnia. Insomniacs with higher physiological arousal have-at least during sleep—an increased sympathetic activity and their vagal tone is decreased (Bonnet \& Arand, 1998; Vgontzas et al., 1998). However, a recent CR study showed no significant differences in HRV during wakefulness between insomniacs and matched healthy sleepers (Varkevisser et al., 2005). WVD exhibit selective DIS, but they do not, as insomniacs do, have problems maintaining sleep; nor do they manifest premature morning awakenings with a resultant reduced total sleep time and daytime sleepiness (Kräuchi et al., 2005). WVD do not show higher hypothalamic-pituitary-adrenal (HPA) axis activity (same basal cortisol level; Kräuchi unpublished data) as often found in patients with insomnia (Buckley \& Schatzberg, 2005). Therefore, a direct effect of DIS on HRV pattern seems rather unlikely.

From a chronobiological viewpoint, WVD exhibit differences in phase of entrainment. Their sleep-wake cycle does not differ from CON, but the circadian system is significantly phase-delayed (Vollenweider et al., 2008). A different phase angle leads to chronic circadian misalignment, which could lead to cardiovascular dysfunction-as found in shiftworkers (Boggild \& Knutsson, 1999). A recent controlled laboratory study simulated the effect of circadian misalignment on the physiological pattern, but found no decrease in the HF band (Scheer et al., 2009). A short-term circadian misalignment may be quite different from chronic circadian misalignment as in our subjects.

\section{What Impact Does Time Have on HRV-Does a Circadian Rhythm Exist?}

Paced-breathing HRV data revealed a linear decrease of vagal activity and a linear increase of sympathovagal balance with elapsed time awake and elapsed time lying in bed. Contradictory findings have been reported for these variables. In some studies with prolonged wakefulness, the output for the vagal branch of the ANS exhibited no decrease (Burgess et al., 1997; Holmes et al., 2002; Vandewalle et al., 2007), whereas other studies revealed an increase (Van Eekelen et al., 2004) or a decrease in vagal activity (Zhong et al., 2005). These different findings could be due to different protocols, such as different duration of time awake, controlled posture or not, or different parameters used for measuring activity of the vagal branch of the ANS. Our data follow those of Zhong et al. (2005), since in protocols where sleep was allowed and subjects were not allowed 
to get up, no withdrawal of the vagal branch (Burgess et al., 1997; Kräuchi et al., 2000) or increase of sympathovagal balance could be established over time (Kräuchi et al., 2000). Thus, recumbency, per se, has no effect on vagal attenuation or of $\mathrm{LF} / \mathrm{HF}$ ratio elevation. Taken together, our results support the hypothesis that vagal activity is responsive to homeostatic sleep pressure and additionally that sleep seems to be crucial for the regeneration of vagal activity and, hence, for a reduction of sympathovagal balance.

Our results are in accordance with other CR studies (Kerkhof et al., 1998; Van Dongen et al., 2001) with respect to the lack of day/night variations in BP during sustained wakefulness. Additionally, we also found a slight increase in DBP over time, which had never been previously reported. A higher DBP indicates a less than maximal dilatation of the arteries, which in turn could be in concert with the withdrawal of $\mathrm{HF}$ power over time (regression analysis: slopes of DBP versus HF power, $r=-.55, p<.05 ; \mathrm{N}=9$ pairs). $\mathrm{BP}$ regulation encompasses the interplay between the central nervous system (CNS), vasoactive molecules, baroreceptor sensitivity, HPA axis activity, and the ANS. Every factor has its own rhythmicity and individual sensitivity (Portaluppi \& Smolensky, 2001). Additionally, sleep has a profound influence on blood pressure. To what extent microsleep occurred in our subjects was not measured.

Breathing rates may provide the underlying reason for the nonexistent circadian rhythm in HF power, which is in contrast to other studies (Burgess et al., 1997; Hilton et al., 2000; Vandewalle et al., 2007; Van Eekelen et al., 2004), particularly when one considers that these rates were the most apparent difference between this and the other study protocols. If breathing is not paced, the rhythmicity of HF power might be due to the circadian rhythm in respiratory control. In fact, the circadian rhythm in respiratory control without sleep is known (Spengler et al., 2000). If paced breathing is applied, respiration rate should be the same at all timepoints, which could abolish the circadian rhythmicity in HF power during prolonged wakefulness. Another distinction between this and the other studies is sex of the subjects. To our knowledge, this is the first study in which all subjects were women and assessed during their luteal phase under CR conditions. It is known that women exhibit a different HRV pattern than men (Antelmi et al., 2004; Bonnemeier et al., 2003; John, 2007; Liao et al., 1995; Umetani et al., 1998), that the HRV pattern differs according to the hormonal cycle (Bai et al., 2009; Sato et al., 1995), and that women live under higher sleep pressure than men (Birchler-Pedross et al., 2009), which all might explain the linear decrease in $\mathrm{HF}$ power over time.

A circadian rhythm was found for LF power. To our knowledge only one study has previously analyzed the LF component of the HRV spectrum with respect to circadian variations in a constant-routine setting, 
and it also found a significant endogenous circadian rhythm (Vandewalle et al., 2007).

Due to the correlated curves for sleepiness and LF/HF ratio $(r=.53$, $p<.05, F=7.29 ; \mathrm{N}=18$ ), one might argue that the ANS regulates against fatigue by an elevated sympathetic branch of the ANS during the night, when sleepiness is highest, seen in elevated LF power and a decrease in the vagal branch of the ANS during sN and on D2. This finding provides evidence that a CR protocol not only unmasks circadian patterns, such as core body temperature, but also induces masking effects on the ANS.

\section{Limitations}

Paced breathing occurred every $2 \mathrm{~h}$ over the CR protocol; therefore, HRV values were analyzed for the same timepoints. In contrast, BP was measured by the cuff method, using a standard sphygmomanometer every hour, not permitting analysis of the BP variability (BPV) for a direct sympathetic influence. In order to estimate the relationship between skin blood flow and activity of the vagal and the sympathetic branches of the ANS in a more conclusive way, different measurements have to be additionally considered in future studies, e.g., BPV, laser Doppler flowmetry, and peripheral arterial tonometry. Respiratory volume was not measured; however, rhythms in the cardiovascular system were comparable with and without control of tidal volume (Cooke et al., 1998). One of the programs used for circadian phase estimation has been validated only for core body temperature or melatonin. To underpin the phases of the HR and HRV indices, a second program with a slightly different approach was used as well. Due to the relative small number of subjects, a larger sample is needed to confirm present data.

\section{CONCLUSION}

WVD manifest lower vagal activity and higher sympathovagal balance. The circadian rhythm of LF power showed a peak during the late night, which suggests that heart activity starts increasing prior to habitual awakening. This finding is seemingly related to the $24-\mathrm{h}$ pattern of cardiac events, which occur most often immediately after awakening. However, reduced vagal and increased sympathovagal balance in WVD indicate an adverse physical constellation, which could be related to myocardial ischemia (Sroka, 2004) and to a higher predisposition for the development of coronary artery disease (Schwartz et al., 1992). Recent studies have shown that VLF is a predictor for myocardial ischemia as well (Bigger et al., 1992; Quintana et al., 1997). This parameter was slightly reduced in WVD. Taken together, WVD might be at a higher 
risk of developing heart failure due to an autonomic imbalance. Additionally the ANS in women seems to follow two strategies to fight against sleepiness. One is based on circadian control, the other based on homeostatic control, and both are reflected by the sympathovagal balance.

\section{ACKNOWLEDGMENTS}

We are very grateful to Prof. Anna Wirz-Justice for helpful comments to the manuscript, Doreen Hartwich for discussion, and Sarah Chellappa and Jakub Späti for corrections. We thank also Claudia Renz, MarieFrance Dattler, and Giovanni Balestrieri for their help in data acquisition and the volunteers for participating. This work was supported by the Swiss National Science Foundation grants 3100A0-102182/1 and 3200B0-116504/1 (to K.K.), the Gottlieb Daimler and Karl Benz Foundation, and the Schwickert-Stiftung.

Declaration of Interest: The authors report no conflicts of interest. The authors alone are responsible for the content and writing of the paper.

\section{REFERENCES}

Akerstedt T, Gillberg M. (1990). Subjective and objective sleepiness in the active individual. Int. J. Neurosci. 52:29-37.

Akselrod S, Gordon D, Ubel FA, Shannon DC, Berger AC, Cohen RJ. (1981). Power spectrum analysis of heart rate fluctuation: a quantitative probe of beat-to-beat cardiovascular control. Science 213:220-222.

Alvarenga MS, Scagliusi FB, Philippi ST. (2008). Changing attitudes, beliefs and feelings towards food in bulimic patients. Arch. Latinoam. Nutr. 58:274-279.

Antelmi I, de Paula RS, Shinzato AR, Peres CA, Mansur AJ, Grupi CJ. (2004). Influence of age, gender, body mass index, and functional capacity on heart rate variability in a cohort of subjects without heart disease. Am. J. Cardiol. 93:381-385.

Bai X, Li J, Zhou L, Li X. (2009). Influence of the menstrual cycle on nonlinear properties of heart rate variability in young women. Am. J. Physiol. Heart Circ. Physiol. 297:H765-H774.

Bigger JT Jr, Fleiss JL, Steinman RC, Rolnitzky LM, Kleiger RE, Rottman JN. (1992). Frequency domain measures of heart period variability and mortality after myocardial infarction. Circulation 85:164-171.

Birchler-Pedross A, Schroder CM, Münch M, Knoblauch V, Blatter K, Schnitzler-Sack C, Wirz-Justice A, Cajochen C. (2009). Subjective well-being is modulated by circadian phase, sleep pressure, age, and gender. J. Biol. Rhythms 24:232-242.

Bleil ME, Gianaros PJ, Jennings JR, Flory JD, Manuck SB. (2008). Trait negative affect: toward an integrated model of understanding psychological risk for impairment in cardiac autonomic function. Psychosom. Med. 70:328-337.

Boggild H, Knutsson A. (1999). Shift work, risk factors and cardiovascular disease. Scand. J. Work Environ. Health 25:85-99.

Bonnemeier H, Richardt G, Potratz J, Wiegand UK, Brandes A, Kluge N, Katus HA. (2003). Circadian profile of cardiac autonomic nervous modulation in healthy subjects: differing effects of aging and gender on heart rate variability. J. Cardiovasc. Electrophysiol. 14:791-799.

Bonnet MH, Arand DL. (1998). Heart rate variability in insomniacs and matched normal sleepers. Psychosom. Med. 60:610-615. 
Brenner IK, Thomas S, Shephard RJ. (1997). Spectral analysis of heart rate variability during heat exposure and repeated exercise. Eur. J. Appl. Physiol. Occup. Physiol. 76:145-156.

Brown EN, Czeisler CA. (1992). The statistical analysis of circadian phase and amplitude in constantroutine core-temperature data. J. Biol. Rhythms 7:177-202.

Bruce-Low SS, Cotterrell D, Jones GE. (2006). Heart rate variability during high ambient heat exposure. Aviat. Space Environ. Med. 77:915-920.

Buckley TM, Schatzberg AF. (2005). On the interactions of the hypothalamic-pituitary-adrenal (HPA) axis and sleep: normal HPA axis activity and circadian rhythm, exemplary sleep disorders. J. Clin. Endocrinol. Metab. 90:3106-3114.

Burgess HJ, Trinder J, Kim Y, Luke D. (1997). Sleep and circadian influences on cardiac autonomic nervous system activity. Am.J. Physiol. 273:H1761-H1768.

Cohen H, Benjamin J, Geva AB, Matar MA, Kaplan Z, Kotler M. (2000). Autonomic dysregulation in panic disorder and in post-traumatic stress disorder: application of power spectrum analysis of heart rate variability at rest and in response to recollection of trauma or panic attacks. Psychiatry Res. 96:1-13.

Cooke WH, Cox JF, Diedrich AM, Taylor JA, Beightol LA, Ames JEIV, Hoag JB, Seidel H, Eckberg DL. (1998). Controlled breathing protocols probe human autonomic cardiovascular rhythms. Am. J. Physiol. 274:H709-H718.

Curran-Everett D. (2000). Multiple comparisons: philosophies and illustrations. Am. J. Physiol. Regul. Integr. Comp. Physiol. 279:R1-R8.

Emre M, Orgül S, Gugleta K, Flammer J. (2004). Ocular blood flow alteration in glaucoma is related to systemic vascular dysregulation. Br. J. Ophthalmol. 88:662-666.

Flammer J, Pache M, Resink T. (2001). Vasospasm, its role in the pathogenesis of diseases with particular reference to the eye. Prog. Retin. Eye Res. 20:319-349.

Fleisher LA, Frank SM, Sessler DI, Cheng C, Matsukawa T, Vannier CA. (1996). Thermoregulation and heart rate variability. Clin. Sci. (Lond) 90:97-103.

Friedman BH, Thayer JF. (1998a). Anxiety and autonomic flexibility: a cardiovascular approach. Biol. Psychol. 47:243-263.

Friedman BH, Thayer JF. (1998b). Autonomic balance revisited: panic anxiety and heart rate variability. J. Psychosom. Res. 44:133-151.

Gasser P, Flammer J. (1991). Blood-cell velocity in the nailfold capillaries of patients with normaltension and high-tension glaucoma. Am.J. Ophthalmol. 111:585-588.

Goldstein S, Zoble RG, Akiyama T, Cohen JD, Lancaster S, Liebson PR, Rapaport E, Goldberg AD, Peters RW, Gillis AM. (1996). Relation of circadian ventricular ectopic activity to cardiac mortality. CAST Investigators. Am. J. Cardiol. 78:881-885.

Gompper B, Vollenweider S, Renz C, Someren E, Wirz-Justice A, Orgül S, Flammer J, Kräuchi K. (2007). Ambulatory measurement of skin temperatures and the sleep-wake-cycle in women with vasospastic syndrome and controls. Sleep 30(Abstr Suppl):A51-A52.

Gupta A, Shetty H. (2005). Circadian variation in stroke-a prospective hospital-based study. Int. J. Clin. Pract. 59:1272-1275.

Guzik P, Piskorski J, Krauze T, Schneider R, Wesseling KH, Wykretowicz A, Wysocki H. (2007). Correlations between the Poincaré plot and conventional heart rate variability parameters assessed during paced breathing. J. Physiol. Sci. 57:63-71.

Hilton MF, Umali MU, Czeisler CA, Wyatt JK, Shea SA. (2000). Endogenous circadian control of the human autonomic nervous system. Comput. Cardiol. 27:197-200.

Holmes AL, Burgess HJ, Dawson D. (2002). Effects of sleep pressure on endogenous cardiac autonomic activity and body temperature. J. Appl. Physiol. 92:2578-2584.

House JR, Tipton MJ. (2002). Using skin temperature gradients or skin heat flux measurements to determine thresholds of vasoconstriction and vasodilatation. Eur. J. Appl. Physiol. 88:141-145.

John Z. (2007). Effect of age and sex on heart rate variability in healthy subjects. J. Manipulative Physiol. Ther. 30:374-379.

Johnsen BH, Thayer JF, Laberg JC, Wormnes B, Raadal M, Skaret E, Kvale G, Berg E. (2003). Attentional and physiological characteristics of patients with dental anxiety. J. Anxiety Disord. $17: 75-87$.

Kerkhof GA, Van Dongen HP, Bobbert AC. (1998). Absence of endogenous circadian rhythmicity in blood pressure? Am. J. Hypertens. 11:373-377. 
Klein E, Cnaani E, Harel T, Braun S, Ben-Haim SA. (1995). Altered heart rate variability in panic disorder patients. Biol. Psychiatry 37:18-24.

Kräuchi K, Wirz-Justice A. (1994). Circadian rhythm of heat production, heart rate, and skin and core temperature under unmasking conditions in men. Am. J. Physiol. 267:R819-R829.

Kräuchi K, Von Arb M, Werth E, Renz C, Wirz-Justice A. (2000). Morning melatonin administration and heart rate variability in healthy young men during 58 hours constant bedrest [abstract]. Sleep 23:A110-A111.

Kräuchi K, Fontana Gasio P, Renz C, Orgül S, Flammer J, Zemp Stutz E. (2005). The Basel survey on sleep behavior and vasospastic syndrome: evidence for an association of sleep onset insomnia with peripheral vasoconstriction [abstract]. Sleep 28:A236-A237.

Liao D, Barnes RW, Chambless LE, Simpson R, Jr, Sorlie P, Heiss G. (1995). Age, race, and sex differences in autonomic cardiac function measured by spectral analysis of heart rate variability-the ARIC study. Atherosclerosis Risk in Communities. Am. J. Cardiol. 76:906-912.

Liu W, Lian Z, Liu Y. (2008). Heart rate variability at different thermal comfort levels. Eur. J. Appl. Physiol. 103:361-366.

Marci CD, Glick DM, Loh R, Dougherty DD. (2007). Autonomic and prefrontal cortex responses to autobiographical recall of emotions. Cogn. Affect. Behav. Neurosci. 7:243-250.

Marsh EE 3rd, Biller J, Adams HP Jr., Marler JR, Hulbert JR, Love BB, Gordon DL. (1990). Circadian variation in onset of acute ischemic stroke. Arch. Neurol. 47:1178-1180.

Mills JN, Minors DS, Waterhouse JM. (1978). Adaptation to abrupt time shifts of the oscillator(s) controlling human circadian rhythms. J. Physiol. 285:455-470.

Muller JE. (1999). Circadian variation in cardiovascular events. Am. J. Hypertens. 12:35S-42S.

Nagashima K, Yoda T, Yagishita T, Taniguchi A, Hosono T, Kanosue K. (2002). Thermal regulation and comfort during a mild-cold exposure in young Japanese women complaining of unusual coldness. J. Appl. Physiol. 92:1029-1035.

Narita K, Murata T, Hamada T, Takahashi T, Omori M, Suganuma N, Yoshida H, Wada Y. (2007). Interactions among higher trait anxiety, sympathetic activity, and endothelial function in the elderly. J. Psychiatr. Res. 41:418-427.

Ottaviani C, Shapiro D, Davydov DM, Goldstein IB, Mills PJ. (2009). The autonomic phenotype of rumination. Int. J. Psychophysiol. 72:267-275.

Pagani M, Lombardi F, Guzzetti S, Rimoldi O, Furlan R, Pizzinelli P, Sandrone G, Malfatto G, Dell'Orto S, Piccaluga E, Turiel M, Baselli G, Cerutti S, Malliani A. (1986). Power spectral analysis of heart rate and arterial pressure variabilities as a marker of sympatho-vagal interaction in man and conscious dog. Circ. Res. 59:178-193.

Pomeranz B, Macaulay RJ, Caudill MA, Kutz I, Adam D, Gordon D, Kilborn KM, Barger A C, Shannon DC, Cohen RJ, Benson H. (1985). Assessment of autonomic function in humans by heart rate spectral analysis. Am. J. Physiol. 248:H151-H153.

Portaluppi F, Smolensky MH. (2001). Circadian rhythm and environmental determinants of blood pressure regulation in normal and hypertension conditions. In White WB (ed.), Blood pressure monitoring in cardiovascular medicine and therapeutics. Totowa, NJ: Humana Press, pp. 79-138.

Portaluppi F, Touitou Y, Smolensky MH. (2008). Ethical and methodological standards for laboratory and medical biological rhythm research. Chronobiol. Int. 25:999-1016.

Quintana M, Storck N, Lindblad LE, Lindvall K, Ericson M. (1997). Heart rate variability as a means of assessing prognosis after acute myocardial infarction. A 3-year follow-up study. Eur. Heart J. 18:789-797.

Rubinstein EH, Sessler DI. (1990). Skin-surface temperature gradients correlate with fingertip blood flow in humans. Anesthesiology 73:541-545.

Sato N, Miyake S, Akatsu J, Kumashiro M. (1995). Power spectral analysis of heart rate variability in healthy young women during the normal menstrual cycle. Psychosom. Med. 57:331-335.

Scheer FA, Hilton MF, Mantzoros CS, Shea SA. (2009). Adverse metabolic and cardiovascular consequences of circadian misalignment. Proc. Natl. Acad. Sci. U.S.A. 106:4453-4458.

Schwartz PJ, La Rovere MT, Vanoli E. (1992). Autonomic nervous system and sudden cardiac death. Experimental basis and clinical observations for post-myocardial infarction risk stratification. Circulation 85:177-191.

Severens NM, Van Marken Lichtenbelt WD, Frijns AJ, Kingma BR, de Mol BA, Van Steenhoven AA. (2010). Measurement of model coefficients of skin sympathetic vasoconstriction. Physiol. Meas. 31:77-93. 
Slinker BK, Glantz SA. (2008). Multiple linear regression: accounting for multiple simultaneous determinants of a continuous dependent variable. Circulation 117:1732-1737.

Spengler CM, Czeisler CA, Shea SA. (2000). An endogenous circadian rhythm of respiratory control in humans. J. Physiol. 526(Pt 3):683-694.

Sroka K. (2004). On the genesis of myocardial ischemia. Z. Kardiol. 93:768-783.

Task Force of the European Society of Cardiology and the North America Society of Pacing Electrophysiology. (1996) Heart rate variability: standards of measurement, physiological interpretation, and clinical use. Eur. Heart J. 17:354-381.

Torsvall L, Akerstedt T. (1980). A diurnal type scale. Construction, consistency and validation in shift work. Scand. J. Work Environ. Health 6:283-290.

Umetani K, Singer DH, McCraty R, Atkinson M. (1998). Twenty-four hour time domain heart rate variability and heart rate: relations to age and gender over nine decades. J. Am. Coll. Cardiol. 31:593-601.

Van Dongen HP, Maislin G, Kerkhof GA. (2001). Repeated assessment of the endogenous 24-hour profile of blood pressure under constant routine. Chronobiol. Int. 18:85-98.

Van Eekelen AP, Houtveen JH, Kerkhof GA. (2004). Circadian variation in base rate measures of cardiac autonomic activity. Eur. J. Appl. Physiol. 93:39-46.

Vandewalle G, Middleton B, Rajaratnam SM, Stone BM, Thorleifsdottir B, Arendt J, Dijk DJ. (2007). Robust circadian rhythm in heart rate and its variability: influence of exogenous melatonin and photoperiod. J. Sleep Res. 16:148-155.

Varkevisser M, Van Dongen HP, Kerkhof GA. (2005). Physiologic indexes in chronic insomnia during a constant routine: evidence for general hyperarousal? Sleep 28:1588-1596.

Vgontzas AN, Constantine T, Edward OB, Constantine AS, Keith Z, Anthony K, Antonio V-B, George PC. (1998). Chronic insomnia and activity of the stress system: a preliminary study. $J$. Psychosom. Res. 45:21-31.

Vollenweider S, Wirz-Justice A, Flammer J, Orgül S, Kräuchi K. (2008). Chronobiological characterization of women with primary vasospastic syndrome: body heat loss capacity in relation to sleep initiation and phase of entrainment. Am. J. Physiol. Regul. Integr. Comp. Physiol. 294:R630-R638.

Von Arb M, Gompper B, Meyer AH, Zemp Stutz E, Orgül S, Flammer J, Kräuchi K. (2009). Relationship between gender role, anger expression, thermal discomfort and sleep onset latency in women. Biopsychosoc. Med. 3:1-7.

Yao Y, Lian Z, Liu W, Shen Q. (2008). Experimental study on physiological responses and thermal comfort under various ambient temperatures. Physiol. Behav. 93:310-321.

Zhong X, Hilton HJ, Gates GJ, Jelic S, Stern Y, Bartels MN, Demeersman RE, Basner RC. (2005). Increased sympathetic and decreased parasympathetic cardiovascular modulation in normal humans with acute sleep deprivation. J. Appl. Physiol. 98:2024-2032.

Zuther P, Gorbey S, Lemmer B. (2009). Chronos-Fit, revised Version 1.06. http://www.ma.uniheidelberg.de/inst/phar/lehre/chrono.html. 